\title{
Disponibilidade de dados abertos para a resiliência às inundações em Curitiba (Paraná)
}

\author{
Murilo Noli $^{\mathrm{a}}{ }^{\oplus}$, Larissa Ferentz ${ }^{\mathrm{b}}{ }^{\circ}$ e Carlos Mello Garcias $^{\mathrm{c}}$ \\ a Pontifícia Universidade Católica do Paraná, Escola de Arquitetura e Design, Programa de Pós- \\ Graduação em Gestão Urbana, Curitiba, PR, Brasil. E-mail: murilonoli @ gmail.com \\ ${ }^{\mathrm{b}}$ Pontifícia Universidade Católica do Paraná, Escola de Arquitetura e Design, Programa de Pós- \\ Graduação em Gestão Urbana, Curitiba, PR, Brasil. E-mail: ferentzengenharia@ gmail.com \\ ${ }^{\text {c }}$ Pontifícia Universidade Católica do Paraná, Escola de Arquitetura e Design, Programa de Pós- \\ Graduação em Gestão Urbana, Curitiba, PR, Brasil. E-mail: carlos.garcias@ pucpr.br
}

Submetido em 1 de março de 2020. Aceito em 4 de setembro de 2020. https://doi.org/10.47235/rmu.v8i2.139

\begin{abstract}
Resumo. A disponibilização de dados atualizados é um desafio enfrentado atualmente pelos gestores urbanos. A partir da lei de acesso à informação, ressaltou-se a importância de tornar dados abertos acessiveis, para que possam ser livremente usados pela população. Ao mesmo tempo, a incidência de desastres nas cidades aumentou nos últimos anos, sendo a produção de dados de risco de desastres uma das funções do governo. Quanto mais detalhada for a identificação dos riscos associados aos perigos naturais, mais eficazes se tornarão as medidas para redução dos impactos. O objetivo deste trabalho é compreender se os dados abertos estão sendo disponibilizados e são suficientes para serem utilizados como instrumento de resiliência às inundações. Para tanto, a aplicação foi realizada na cidade de Curitiba, em três etapas: (1) busca de arquivos no portal de dados abertos da prefeitura; (2) busca de arquivos não sistematizados nas páginas oficiais do município; e (3) entrevista com atores sociais a respeito da importância do acesso aos dados na compreensão de riscos. Os resultados mostraram que $77 \%$ dos 84 itens investigados apresentaram arquivos abertos. Todos os atores sociais afirmam não receber quaisquer incentivos por parte do governo em utilizar dados abertos, mas consideram a disponibilização deles muito importante.
\end{abstract}

Palavras-chave. riscos e desastres, gestão municipal, Paraná.

\section{Introdução}

A busca por cidades e sociedades resilientes a desastres vem exigindo cada vez mais que os formuladores de políticas e os indivíduos tenham acesso a dados e informações atualizadas, detalhadas e de qualidade do ambiente construído, de maneira especial sobre como os riscos associados aos perigos naturais interagem com a sociedade. Com esse acesso é possível empregar medidas eficazes, como escolher o local mais seguro para instalação de uma escola ou unidade básica de saúde e medidas de proteção
(Calkins, 2015). Tal fato ganha importância à medida que os riscos, impactos e prejuízos de todas as ordens e de grande monta decorrentes dos eventos extremos e desastres serão evitados ou reduzidos por meio do uso de informações, serviços geoespaciais e dados abertos (UNDRR, 2019).

Uma das funções de um governo é produzir dados de risco de desastres relevantes para o seu território, os quais podem ser realizados em sinergia com os demais atores urbanos, como o setor privado, universidades, organizações não-governamentais e 
sociedade. Há inúmeros dados que podem ser empregados como subsídio para o desenvolvimento de estratégias e ações de redução de riscos de desastres. Por exemplo, implementação dos parâmetros de zoneamento e de uso e ocupação do solo; caracterização da região quanto os tipos de solo, bacias hidrográficas e vegetação; monitoramento das infraestruturas críticas; identificação das cotas de inundação; mapeamento das áreas de risco e vulnerabilidade social; dentre outros. Quando eles são inexistentes, ou com restrições, podem gerar consequências para a ciência, $o$ planejamento e a sociedade. Além disso, sem o conhecimento dos usuários de como analisar e aplicar esses dados, os atores urbanos, sobretudo os expostos aos riscos, podem não conseguir gerenciar com eficácia suas vulnerabilidades (Goëta, 2012). Logo, o desconhecimento pode dificultar ou até mesmo impedir a redução de riscos de desastres e impedir a promoção da resiliência aos perigos (Li et al., 2019; Murnane et al., 2019).

Baseado nisto, os governos necessitam de um conjunto de práticas para coletar e conectar os dados sobre riscos, exposição e vulnerabilidade, os quais se encontram, em muitos casos, "fragmentados, incompletos, desatualizados e às vezes imprecisos" (Kassen, 2013, p.12), aos usuários que precisam deles. Logo, a aplicação dos conceitos de dados abertos pode aumentar a qualidade e a disponibilidade de dados e informações, avaliação e modelagem de risco, relatórios e políticas baseadas em evidências, já que consistem em uma das formas menos restritivas de compartilhamento de dados (Serwadda et al., 2018). Ou seja, a democratização dos dados possibilita a construção de uma compreensão coletiva e mais profunda do risco e perspectivas de alta qualidade das próprias comunidades, através do compartilhamento de informações sobre os perigos, exposição, vulnerabilidade e riscos, e do envolvimento dos indivíduos na construção de cidade resilientes a desastres (Kassen, 2013; UNISDR, 2019).

Além do mais, a disponibilização dos dados abertos vai ao encontro do bem-estar e segurança que podem resultar à população. De um lado, eles podem auxiliar a governança pública quanto seu fortalecimento, já que utiliza os princípios de transparência, integridade e prestação de contas (Silva \& Galvão 2018). E, por outro lado, possibilitam a expansão da ciência por meio da academia e pesquisa, visando a solução de problemas e novas perguntas a serem respondidas.

Nessa circunstância, e na necessidade de fortalecer a gestão de riscos e desastres e, por conseguinte, a construção de cidades resilientes, o presente trabalho tem como objetivo central responder as seguintes questões: Os dados abertos estão sendo disponibilizados e são suficientes para possibilitar a resiliência às inundações? Qual a influência dos dados abertos para a promoção da resiliência?

Para tanto, adotou-se como recorte espacial a cidade de Curitiba. Embora ela tenha recebido diversos títulos-slogans que a ressaltam positivamente como "exemplo de planejamento urbano" (1970), "capital de primeiro mundo" (1980), "capital ecológica" (1990) e "capital social" (2000), a realidade a evidencia de modo flagrante com condições de expressiva vulnerabilidade socioambiental e ocorrência de eventos hidrometeorológicos, sobretudo inundações e alagamentos (Zanella, 2006).

O presente artigo foi estruturado, primeiramente, pela revisão dos conceitos sobre dados abertos e resiliência ao risco de desastres. Em seguida, será apresentada a metodologia de pesquisa com vistas à busca de arquivos em formatos aberto e geoespaciais, além da definição do questionário a ser aplicado. O texto será finalizado com os resultados e discussões, apresentados pelos seguintes tópicos de análise: Dados de base; Exposição; Perigo; Vulnerabilidade; e Risco.

\section{Dados abertos}

Os dados abertos consistem em dados digitais brutos que são disponibilizados com características técnicas e legais necessárias, sobretudo pelo poder público, para que sejam livremente usados, reutilizados e redistribuídos por qualquer pessoa, a qualquer hora, em qualquer lugar (Murnane et al., 2019). Esse processo possibilita que as pessoas possam navegar, explorar e aprender 
a buscar questões únicas ou comuns. Com a disponibilização dos dados e a possibilidade de troca, coprodução e avaliação daqueles que são potencialmente afetados como sendo parte fundamental do processo, a resiliência pode ser atingida com êxito (Landry et al., 2016).

Esse movimento representa uma mudança de paradigma. Historicamente, os governos tratavam os dados de diversas formas, mas tinham como ponto comum a produção e distribuição de dados em formato impresso (Johnson et al., 2015). No século XX, os dados eram difíceis de obter, divididos em fragmentos em vários ministérios e caros para remontar. $\mathrm{O}$ advento das tecnologias reduziu o custo da comunicação em ordens de magnitude, representando uma mudança em quem pode acessar os dados oficiais, e sob quais termos (Janssen, Charalabidis, \& Zuiderwijk, 2012). A World Wide Web, por exemplo, conecta não apenas documentos, mas conjuntos de dados entre si. Logo, o custo de compartilhar esses dados está se aproximando de zero e as barreiras à comunicação intraorganizacional são agora sociais e não técnicas e econômicas (Robinson \& Johnson, 2016).

Entretanto, nem todos os dados podem ser considerados como abertos. Os dados fornecidos pelas diferentes esferas do governo brasileiro comumente estão nos formatos XLS, PDF e HTML, chegando a atingir $90 \%$ de disponibilidade em determinados municípios, todavia nem todos são acessíveis por máquinas e estão em um formato não proprietário (Correa et al., 2014). Embora haja uma discussão a respeito de quais dados devem ser considerados como abertos (Santana \& Rodrigues, 2013), há um consenso de que os tabulares ou de texto fornecidos com as extensões CSV, XML, JSON e ODF ou geoespaciais em formato raster e matricial (como GeoTIFF e shapefile, respectivamente) podem ser considerados como abertos (OpenDRI, 2019).

Como forma de torná-los abertos, pode-se empregar duas abordagens: disponibilizar os dados em um site visando facilitar o seu uso - mais comum - ou desenvolver uma estrutura para sustentar o modo mais complexo e contínuo de divulgação. $\mathrm{O}$ desenvolvimento de estrutura é um método recente e consiste na criação de uma política de dados abertos, visando determinar as bases para a continuidade de acesso aos dados, fornecendo informações abertas, transparentes e de alta qualidade, e possibilitando uma melhor compreensão sobre eles e garantindo o acesso a tipos específicos de dados (Li et al., 2019). Por exemplo, os processos e especificações que promovam a eficácia e transparência das plataformas desenvolvidas. As especificações incluem o registro dos metadados, que preservam informações importantes dos dados, tais como título, resumo, data, responsável (is), fontes e métodos de coleta utilizados, padronização da temporalidade da informação, extensão geográfica, tipo de representação espacial, entre outros, como as promulgadas pela ET-EDGV (Especificação Técnica para Estruturação de Dados Geoespaciais Vetoriais), do Exército Brasileiro.

\section{Resiliência ao risco de desastres}

A sociedade contemporânea, sobretudo a que reside em áreas urbanas, vive marcada pelo prenúncio do risco, de desordens, incoerências e pela exposição diária, devido ao célere crescimento populacional, das desigualdades sociais e da deterioração do meio ambiente, como efeito do desenvolvimento técnico-industrial (Beck, 1992). A matriz conceitual que emerge desses estudos é composta pelo uso das palavras perigo (hazard), risco, vulnerabilidade, exposição, resiliência, entre outros. O risco é compreendido como algo ligado à projeção do futuro, é a possibilidade, a chance estatística da ocorrência de um episódio, cujas consequências provocam impactos negativos em um espaço (UNDRR, 2019). Já o perigo delimita eventos com potencial de causar danos, fenômenos naturais ou processos induzidos pelo ser humano, que causam a ruptura de uma sequência e ocasionam prejuízos à sociedade (Hogan e Marandola Jr., 2009). Dessa forma, o mais importante na magnitude do risco não é o tipo de evento, mas as circunstâncias sociais, econômicas e ambientais, sobretudo a vulnerabilidade (Mehmood, 2015).

Nesse caso, compreender a vulnerabilidade dentro das cidades - grupos populacionais que possuem habilidades, habitações e 
condições socioeconômicas diferentes desempenha um papel importante em tornar uma cidade mais resiliente (Landry et al., 2016). Uma pergunta recorrente e fundamental em estudos dessa natureza é: "vulnerabilidade a quê?". Não se pode esquecer da seguinte questão: onde e quem está vulnerável? Geralmente é assinalada a população que possui baixo poder aquisitivo, pois, numa sociedade que se reproduz por meio da produção de um espaço injusto e desigual, se veem forçados a residir em áreas inadequadas, como os assentamentos precários, cujas edificações são instaladas de forma imprópria e apresentam fragilidades. Logo, ser vulnerável é estar exposto a um perigo, é exibir fragilidade diante deste e "reduzir a vulnerabilidade não consiste em tentar reduzir a frequência do hazard, [...] mas em diminuir os efeitos possíveis da crise por meio do conhecimento dos processos e pela instalação de dispositivos adequados" (Veyret \& Richemond, 2007, p. 43).

$\mathrm{O}$ conceito de resiliência emerge neste contexto como um termo derivado da palavra latina "resiliere", que significa retornar a um estado anterior, voltar, recuar (Hosseini, Barker, \& Ramirez-Marquez, 2015). Ela está associada à resistência ao estresse. Os sistemas, quando expostos a situações adversas variam em sua "resistência" impacto relacionado a perturbação exógena e na sua "recuperação" que captura os processos endógenos para o sistema voltar ao estado de equilíbrio ou a melhores condições (Sharifi et al., 2017). Dentre os elementos que o caracteriza, destaca-se a robustez; redundância; diversidade; colaboração; integração; inclusão; igualdade; mecanismos de interatividade e feedback;

descentralização; transparência; flexibilidade; integração sócio ecológica; eficiência; melhoria da capacidade adaptativa; previsibilidade; e planejamento (Tyler \& Moench, 2012). Ainda, a aprendizagem, inovação e governança adaptável (Leichenko, 2011).

No recorte temático abordado neste trabalho, a resiliência pode ser definida como a capacidade que os atores e o sistema urbano possuem para sobreviver, se recuperar, se adaptar e crescer rapidamente perante os impactos decorrentes de um desastre e manter a continuidade de suas atividades cotidianas (Christensen, Lewis \& Armesto, 2016). Esta definição reconhece a importância de tomar medidas e reforçar e fortalecer as capacidades e estruturas prédesastre existentes durante as diferentes fases da gestão do risco de desastres e enfatiza a importância da aprendizagem e adaptação (Sharif, 2019). Logo, é adequado para analisar sistemas urbanos, como sistemas dinâmicos que estão expostos a uma ampla gama de riscos e constantemente passam por processos de evolução e transformação (Sharifi \& Yamagata, 2016). Acrescenta-se a isso a expressão "resiliência social". Ela pode ser compreendida como sendo a capacidade de um grupo ou comunidade, mantendo sua coesão, de se organizar para se adaptar às mudanças, aos riscos e às carências; é o esforço coletivo para melhorar o cotidiano, concentrando-se na solidariedade e tessitura diária dos laços sociais (Farias, 2017).

Desse modo, a busca pelo desenvolvimento sustentável e pela resiliência é uma oportunidade para repensar a forma como se está lidando com a prevenção de desastres, sejam eles naturais ou tecnológicos, uma vez que eles "tornam-se uma adaptação às 'externalidades' do desenvolvimento da sociedade moderna e não uma transformação frente às causas produtoras do risco" (Sulaiman \& Aledo, 2016, p.4). Ou seja, possibilita ao município alcançar um desenvolvimento contínuo e sustentável através da integração entre todos os atores do território, de modo que não apenas os sistemas sejam resilientes, mas que todos os cidadãos também se tornem, resultando em maior preparação, organização, adaptação, recuperação e reconstrução na ocorrência de desastres (Folke et al., 2002). Portanto, uma cidade resiliente é considerada "menos vulnerável e assume uma melhor preparação para lidar com a mudança, com a complexidade dos riscos existentes, com crises e perturbações múltiplas, evitando disrupções e colapsos, como consequência de um desastre" (Carvalho et al., 2013, p. 442).

Estas características são essenciais no combate às inundações. Com as altas taxas de urbanização sofrida nos últimos anos em proximidade com os rios, a frequência destes eventos tem aumentado substancialmente nas cidades. Enquanto no mundo, as inundações representam $42 \%$ dos desastres, no Brasil, 
elas chegam a 33\% das ocorrências, sendo as regiões Sudeste, Nordeste e Sul as mais afetadas por elas (Freitas et al., 2014).

No estado do Paraná, são mais de 700 casos registrados de inundação entre 1980 e o início de maio de 2020. Estes desastres afetaram 280 mil pessoas, mataram $32 \mathrm{e}$ resultaram em 385,2 milhões de reais de prejuízo econômico (BI-CEPDEC, 2020).

\section{Curitiba e as inundações}

A problemática socioambiental urbana em Curitiba está envolvida de múltiplas maneiras com os impactos derivados do seu processo de urbanização. A sua área e seu entorno imediato constituem um cenário que explicita essa problemática de modo significativo, sobretudo os problemas relacionados às inundações. Elas ocorrem quando o nível de vazão do curso d'água excede a sua capacidade de descarga, extravasando a água para as margens do leito fluvial. Em Curitiba, esse fenômeno configura-se como processo histórico que permeia a construção da cidade, cuja expansão ocorreu nos vales e confluências dos rios (Geissler \& Loch, 2004). Seus impactos agravaram-se com o rápido e desordenado processo de urbanização, que possibilitou a ocupação de áreas com elevada fragilidade ambiental e destituídas de infraestrutura básica, como saneamento e eletricidade, gerando áreas de risco (Mendonça et al., 2013).

De forma geral, esse problema era restrito a área central de Curitiba no início e meados do século XX, resultando em intervenções constituídas na ótica da contenção de inundações e de seus impactos sobre a sociedade e da reprodução socioespacial de parte da cidade, como a criação de parques e projetos de engenharia hidráulica e sanitária (Mendonça et al., 2013). Mas tais obras ignoraram as verdadeiras causas dos problemas e transferiram os efeitos para a jusante (Geisller \& Loch, 2004). Atrelado a isso, a expansão urbana e a ocupação das áreas pericentrais e periféricas, caracterizadas pela alta fragilidade ambiental e ausência de infraestruturas e planejamento adequado no que se refere à impermeabilização do solo e o escoamento das águas das chuvas, geraram inundações, doenças transmitidas pelo espraiamento das águas, perdas materiais e vítimas humanas, especialmente a partir da década de 1950 (COMEC, 2006).

Atualmente, Curitiba contém 59 áreas de risco a alagamentos e 19 a inundações, compreendendo, juntos, 18.304 residências e 96.250 pessoas passíveis de serem atingidas (CEPDEC, 2019). Do ponto de vista espacial, a maior parcela dessa população está nas regionais do Boqueirão (44.991), CIC (20.900) e Boa Vista (11.659) (CEPDEC, 2019).

\section{Metodologia}

Com a finalidade de avaliar os dados abertos como um instrumento de resiliência em face à ocorrência de inundações, identificando quais secretarias disponibilizam tais dados, a abordagem quali-quantitativa mostra-se mais adequada, tendo em vista que se almeja compreender o fenômeno analisado dentro do seu contexto (social, institucional e organizacional) (Gil, 2019). Para tanto, adotou-se como recorte espacial a cidade de Curitiba. Ela apresenta o maior quantitativo de ocorrências de alagamentos e inundações no Paraná entre 1980 e 2018 (CEPDEC, 2019), e dispõe de uma política de dados abertos (Decreto $\mathrm{n}^{\circ}$ 1135/2012 e Lei $\left.\mathrm{n}^{\circ} .14422 / 2014\right)$.

Os procedimentos metodológicos empregados foram subdivididos em três etapas: (1) Busca de arquivos em formatos abertos (dados tabulares - .CSV, .XML, JSON, .ODF.) e dados geoespaciais (.SHP matricial, GEOTIFF - raster) no portal de dados abertos da prefeitura de Curitiba; (2) Procura de arquivos não sistematizados nas páginas oficiais das secretarias do município; e (3) Aplicação de questionários com atores sociais moradores da cidade de Curitiba, a respeito da importância de ter acesso a tais dados na compreensão dos riscos.

Em um primeiro momento, buscou-se verificar as características legais já determinadas sobre os dados abertos em Curitiba, indícios de institucionalização do tema, passando a ter maior estabilidade em face de trocas de gestão e de alterações de conjunturas políticas, e sob qual área da administração a política de dados abertos encontra-se vinculada.

Também foi verificado se o governo municipal possui mecanismos de incentivo 
ao uso dos dados disponibilizados.

Posteriormente, há um aprofundamento em relação aos dados gerais disponibilizados pelo poder público para os demais atores sociais. Para a análise do portal de dados abertos e dos sítios das secretarias foi elaborado um quadro de avaliação subdividido em 19 pontos principais, as quais pertencem às dimensões de classificação dos dados, indicadores e critérios de dados abertos (Quadro 1).

Quadro 1. Categorias de dados, critérios e indicadores (fonte: OpenIndex, 2019).

\begin{tabular}{|c|c|c|}
\hline Dimensão & \multicolumn{2}{|c|}{ Tópico analisado } \\
\hline \multirow{3}{*}{ Classificação dos dados } & Dados de base & Perigo \\
\hline & Exposição & Vulnerabilidade \\
\hline & Risco & \\
\hline \multirow{5}{*}{ Critérios de dados abertos } & Os dados existem? & $\begin{array}{l}\text { Os dados estão disponíveis em } \\
\text { formato digital? }\end{array}$ \\
\hline & $\begin{array}{c}\text { Os dados estão disponíveis em } \\
\text { massa? }\end{array}$ & $\begin{array}{l}\text { Os metadados estão disponíveis } \\
\text { online? }\end{array}$ \\
\hline & Os dados estão disponíveis online? & $\begin{array}{c}\text { Os dados são legíveis por máquina e } \\
\text { reutilizáveis? }\end{array}$ \\
\hline & $\begin{array}{l}\text { Os dados estão disponíveis } \\
\text { publicamente? }\end{array}$ & $\begin{array}{l}\text { Os dados estão disponíveis } \\
\text { gratuitamente }\end{array}$ \\
\hline & $\begin{array}{l}\text { Os dados são licenciados } \\
\text { abertamente? }\end{array}$ & $\begin{array}{l}\text { Os dados não são fornecidos em } \\
\text { tempo hábil e atualizado? }\end{array}$ \\
\hline \multirow{2}{*}{ Indicadores } & Aberto & Restrito \\
\hline & Fechado & Ausência \\
\hline
\end{tabular}

Na classificação dos dados, empregou-se um conjunto de informações principais para cada categoria, que variam desde a identificação de edifícios, infraestruturas críticas e população (Exposição), limites administrativos, modelo digital de elevação e corpos hídricos (Dados de base), medidas de proteção às inundações, mapas de risco às inundações de alagamentos (Perigo), curvas de vulnerabilidade dos edifícios, vulnerabilidade das atividades econômicas e da população (Vulnerabilidade), até aos indicadores de risco (Quadro 2).

No caso dos indicadores, empregado para avaliar em que medida um determinado conjunto de dados está aberto, considerou-se as seguintes condições:

- $\quad$ se existem (podem estar em qualquer formato - em papel ou digital, offline ou online);

- se estão em formato digital ou se estão disponíveis somente em papel;

- $\quad$ se eles são públicos (isso não exige que ele esteja disponível gratuitamente on-line, mas que alguém fora do governo possa acessá-lo de alguma forma);

- $\quad$ se estão disponíveis online

- $\quad$ se os metadados estão disponíveis online;

- $\quad$ se estão disponíveis em massa (se todo o conjunto de dados pode ser baixado de uma só vez ou acessado facilmente);

- $\quad$ se estão disponíveis gratuitamente ou se há uma cobrança por seu acesso;

- se são licenciados abertamente; e

- $\quad$ se estão atualizados e são oportunos - ou desatualizados.

Adota-se a definição de dados abertos, restritos, fechados ou ausentes estabelecida pela OpenDRI (2019), para quem o primeiro é considerado como aberto quando todas as perguntas acima forem respondidas afirmativamente; o segundo se existir e estiver disponível publicamente, mas pelo menos uma pergunta sobre restrições legais, técnicas ou de custo é respondida não; o terceiro se não estiver disponível 
publicamente; e o último se não existir o dado.

A partir da definição do conjunto de dados sobre risco de inundações, foi realizada a busca de 84 itens e subitens, subdivididos entre as categorias dos grupos de Exposição, Dados de base, Perigo, Vulnerabilidade e Risco. Em primeiro momento, a pesquisa foi realizada no Portal de Dados Abertos de Curitiba.

Após a instituição da Lei $\mathrm{n}^{\circ} 12.527$, de 18 de novembro de 2011, sobre o acesso à informação pública, a Prefeitura Municipal de Curitiba disponibilizou o sítio web "Portal de Dados Abertos" visando a transparência e a participação da população. Nele são apresentados documentos, dados e informações governamentais de domínio público para acesso e utilização da sociedade. Dentre os grupos presentes neste domínio estão: abastecimento, administração pública, cultura, empreendedorismo, financeiro, governo municipal, habitação, legislação, pesquisa e planejamento, recursos humanos, saúde, segurança, transporte e turismo.

Quadro 2. Conjunto de dados sobre o risco de inundação (fonte: elaborado pelos autores).

\begin{tabular}{|c|c|c|c|}
\hline \multicolumn{4}{|c|}{ Dados de base } \\
\hline Limites administrativos & Imagens aéreas & Pavimentação & Tipo de solo \\
\hline Topografia e orientação & Altimetria & Hipsometria & Declividade \\
\hline Cobertura vegetal & Corpos d'água & Bacias hidrográficas & Rede hidrográfica \\
\hline Mapa geomorfológico & Arruamento & Área urbanizada & Áreas impermeáveis \\
\hline $\begin{array}{c}\text { Modelo digital de } \\
\text { elevação }\end{array}$ & & & \\
\hline \multicolumn{4}{|c|}{ Exposição } \\
\hline Zoneamento & Uso e ocupação do solo & Cobertura da terra & Loteamentos \\
\hline População & Domicílio & Atividades econômicas & Registro de empresas \\
\hline $\begin{array}{l}\text { Edifícios e ativos } \\
\text { tecnológicos }\end{array}$ & $\begin{array}{l}\text { Equipamentos } \\
\text { municipais }\end{array}$ & $\begin{array}{l}\text { Infraestrutura } \\
\text { administrativa }\end{array}$ & $\begin{array}{c}\text { Patrimônio histórico e } \\
\text { cultural }\end{array}$ \\
\hline $\begin{array}{l}\text { Equipamentos } \\
\text { religiosos }\end{array}$ & $\begin{array}{l}\text { Abastecimento } \\
\text { alimentar }\end{array}$ & Infraestrutura turística & $\begin{array}{c}\text { Equipamentos } \\
\text { esportivos }\end{array}$ \\
\hline Infraestrutura crítica & Áreas verdes e lazer & Áreas de risco & Áreas irregulares \\
\hline Vazios Urbanos & Serviços de emergência & Segurança & Fauna \\
\hline \multicolumn{4}{|c|}{ Perigo } \\
\hline $\begin{array}{l}\text { Mapas de risco de } \\
\text { inundação }\end{array}$ & $\begin{array}{l}\text { Medidas de proteção } \\
\text { contra inundação }\end{array}$ & $\begin{array}{l}\text { Registros de eventos de } \\
\text { risco }\end{array}$ & $\begin{array}{l}\text { Avisos/alertas de } \\
\text { inundação }\end{array}$ \\
\hline Dados hidrológicos & Áreas inundáveis & Cotas de inundação & Dados meteorológicos \\
\hline \multicolumn{4}{|c|}{ Vulnerabilidade } \\
\hline Ativos agregados & $\begin{array}{l}\text { Tipo de cobertura } \\
\text { territorial }\end{array}$ & Infraestrutura crítica & População \\
\hline Atividades econômicas & Edifício & Áreas de risco & Equipamentos \\
\hline \multicolumn{4}{|c|}{ Risco } \\
\hline \multicolumn{4}{|c|}{ Indicadores de risco de estudos anteriores } \\
\hline
\end{tabular}

Após a busca no Portal de Dados Abertos, a pesquisa deu continuidade a partir de sites oficiais da prefeitura municipal de Curitiba e correlatos, e posteriormente, em mecanismos de busca.

Não obstante, como forma de compreender a relação da população com o uso de dados abertos, foi realizada a aplicação de 30 entrevistas. Além da caracterização dos participantes, 10 pontos principais foram questionados:

1. Recebimento de algum tipo de informação sobre os riscos de desastres que está sujeito na cidade de Curitiba;

2. Meios de comunicação em que essas informações são recebidas; 
3. Necessidade pela busca de informações sobre riscos ou sobre algum desastre específico nos sites oficiais de Curitiba;

4. Facilidade de acesso para encontrar as informações;

5. Importância da divulgação de informação sobre os riscos para a população;

6. Conhecimento sobre dados abertos;

7. Importância da disponibilidade de dados abertos para que a população possa desenvolver trabalhos e pesquisas;

8. Existência de um portal da prefeitura exclusivo para disponibilizar dados abertos;

9. Possibilidade de encontrar informações de todas as áreas setoriais que pertencem a prefeitura no formato aberto;

10. Incentivo ao uso de dados abertos pelos gestores do município.

A descrição das dimensões e dos questionários aplicados é apresentada a seguir.

\section{Resultados e discussões}

\section{Exposição}

A dimensão de exposição é formada a partir de 23 itens e 39 subitens. Dentre sua composição, $92 \%$ dos tópicos apresentaram dados abertos.

No zoneamento foi realizada a busca por informações sobre a divisão do território, incluindo o sistema viário, a topografia e a infraestrutura existente, por meio de zonas e setores de uso e ocupação do solo e adensamentos diferenciados. Neste quesito foram encontrados quatro tipos de arquivos abertos (.SBN, .SBX, .DWG e .DBF).

Para o uso e ocupação do solo, buscou-se a reprodução social no plano do espaço urbano. Ou seja, a maneira pela qual as edificações podem ocupar o terreno urbano. Não foram encontradas informações abertas exclusivas sobre Uso e Ocupação do Solo, mas arquivos .PDF e .JPEG estão disponíveis. No Brasil, a elaboração de mapas desta temática é realizada em conjunto ao Zoneamento. Desta forma, os mapeamentos disponíveis em regime aberto são de Zoneamento e Uso e Ocupação do Solo.

A cobertura da terra deve apresentar a cobertura do solo e os tipos de uso da terra, como por exemplo, grama, asfalto, árvores, água, áreas urbanas e/ou agrícolas. Dentre os formatos disponíveis estão .CSV, .JSON, .SHP, .KML e .HTML. O item Loteamentos é subdividido em Cemitérios e Habitações Populares. Ambos possuem disponibilidade de arquivos em .CSV, .SBN, .SBX, .DWG e .DBF.

Dentre os subitens da População estão Distribuição populacional, a partir de dados censitários, desagregados no nível administrativo mais baixo (setor censitário); Rendimento médio; Taxa de emprego; e População vulnerável (idosos, crianças e doentes). Todos possuem dados abertos nos formatos .CSV e DWG. O subitem Pessoas com deficiência apresenta apenas informações em .CSV.

As atividades econômicas consideram as estatísticas considerando o PIB. O registro de empresas considera lista de empresas registradas, incluindo seus endereços e setor econômico. Os edifícios e ativos tecnológicos apresentam como subitens o número e as características dos edifícios, na unidade administrativa mais detalhada ou em uma base por prédio e o tipo de uso (residencial, comercial, industrial). Por fim, os equipamentos municipais consideram a classificação de restaurantes, feiras, mercados, dentre outros. Todos estes itens apresentaram dados abertos em formato .CSV.

A infraestrutura crítica é a mais extensa da presente dimensão. Possui um total de 10 subitens. As infraestruturas que fornecem serviços essenciais para as pessoas, redes de telecomunicações, e pontes e trincheiras possuem arquivo aberto apenas em .CSV. Os centros sociais (CRAS e CATI), rua da cidadania, centro de referência da mulher; instalações de educação (escolas, faróis, CMEI, CMAE, escolas estadual, federal, municipal e particular, faculdades, universidade); instalações de saúde (hospitais, clínicas, postos, asilos), unidade municipal de saúde, centro de urgência e 
centro médico, instituto de medicina, clínica de fraturas, casa de saúde, instituição de longa permanência, farmácia, clínica odontológica; sistema viário, ferroviário e rodoviário, cicloviário, estacionamentos; e equipamentos de transporte/terminais (estações tubo), rede integrada de transporte, apresentam cinco tipos de dados abertos (.CSV, .SBN, .SBX, .DWG e .DBF). Em relação a energia, água, esgoto, ETA/ETE, serviços de coleta e gestão de resíduos, podem ser consultados arquivos em .CSV e .SVG. Não foram encontras informações abertas, fechadas ou restritas sobre postos de gasolina.

A análise do patrimônio histórico e cultural buscou por informações sobre museu, teatro, cinema, espaços expositivos, espaços de leitura, clube de xadrez, memorial, centro cultura, biblioteca, auditório. Da infraestrutura administrativa por bancos, cartório, correio. Do abastecimento alimentar por mercado, sacolão, armazém da família, hortas comunitárias, cambio verde. Da infraestrutura turística por hotéis, pensões, linha turismo, taxi. Dos equipamentos religiosos por igreja e mesquita. Das áreas de risco em relação aos fenômenos; e da segurança e serviços de emergência por delegacias, postos policiais e de bombeiros. Todos os itens possuem dados abertos em formato .CVS.

Os equipamentos esportivos (infraestruturas que fornecem serviços essenciais para as pessoas. Incluem estádios, ginásios, piscinas, academia ao ar livre, unidades desportivas, clube da gente); áreas verdes e de lazer (Áreas Verdes, Praças, parques, jardins, bosques, jardinete, RPPN, Estação de Sustentabilidade); áreas irregulares (residências precárias, residências irregulares, ocupações irregulares regularizadas; aglomerados subnormais); e vazios urbanos (espaços não construídos e não qualificados como áreas livres no interior do perímetro urbano da cidade), estão disponíveis em .CSV, .SBN, .SBX, .DWG e .DBF. Por fim, quanto ao item Fauna, que busca por informação de animais domésticos, zoológicos e abrigos, não foram encontrados dados abertos, fechados ou restritos.

\section{Dados de Base}

A dimensão de dados de base é formada a partir de 17 itens e 21 subitens. Dentre sua composição, $67 \%$ dos tópicos apresentaram dados abertos.

$\mathrm{O}$ item limites administrativos é composto por três subitens. Os limites oficiais das regionais e limites oficiais dos bairros apresentaram dados abertos nos formatos .SBN, .SBX, .DWG e .DBF. Já os Limites oficiais do município não foram encontrados de maneira isolada. Apenas por dados fechados JPEG ou mapa interativo diretamente no site de busca. Dados de topografia e orientação, Declividade e Hipsometria também só foram encontrados em modo fechado, nos formatos PDF ou mapa interativo.

O tipo de solo é composto pelos itens de permeabilidade e mapa de caracterização geológica do tipo de solo. No primeiro caso só foi encontrado material em modo fechado (PDF), enquanto no segundo caso foram encontrados cinco formatos diferentes de dados abertos (.CSV, .JSON, .SHP, .KML e .HTML). O modelo de elevação digital descreve a superfície do terreno e abrange todas as áreas propensas a inundações. Foram encontrados dados abertos nos formatos de .ADF, .CSV, .JSON, .SHP, .KML e .HTML. Nestes formatos também foram identificados Compartimentos geomorfológicos e Formas geomorfológicas, subitens pertencentes a mapa geomorfológico.

Imagens aéreas, como visão vertical ou ortofoto; altimetria, curvas de nível; cobertura vegetal, tipos ou formas de vegetação de origem natural ou plantada que recobrem uma determinada área ou terreno; corpos d'água, mapa dos principais corpos de água (rede fluvial, lagos, litoral); rede hidrográfica; e arruamento, foram encontrados em quatro tipos de arquivo aberto (.SBN, .SBX, .DWG e .DBF). Enquanto os limites de bacias hidrográficas, divisões topográficas que delineiam áreas onde o escoamento de águas superficiais drena para um corpo comum de águas superficiais e a Pavimentação, foram encontrados apenas um tipo de arquivo cada (.SHP. para o primeiro e .CVS para o segundo). 
Por fim, os itens de área urbanizada e de áreas impermeáveis só foram identificados em dados fechados (PDF).

\section{Perigo}

A dimensão de perigo é formada a partir de 8 itens e mais 10 subitens. Dentre sua composição, $40 \%$ dos tópicos apresentaram dados abertos.

Os mapas de risco de inundação descrevem as áreas geográficas que são propensas a inundações durante um determinado período de retorno (normalmente entre 2 e 1000 anos). Juntamente a eles estão as Áreas Inundáveis. Não foram identificados dados abertos para estes itens, apenas fechados em formato JPEG e PDF.

Os dados meteorológicos (dados do medidor histórico de precipitação, temperatura e vento) e os dados hidrológicos (subitens de vazão e de Níveis históricos de água e descargas de rio a partir de estações de bitola nos principais ramos do rio), foram encontrados em formatos .XLS e .TXT. Além destes, os registros históricos de eventos de risco, incluindo o tipo, a intensidade, a pegada, a descrição, a localização e a data dos eventos de risco, foram encontrados em .CVS.

$\mathrm{O}$ item cotas de inundação, que se refere ao tempo de retorno e profundidade de submersão das áreas atingidas, não foi encontrado em nenhum tipo de formato (aberto, fechado ou restrito). Já as medidas de proteção contra inundações (principais estruturas de defesa contra inundações que refletem as mais recentes construções de mitigação de inundações) e avisos/alertas de inundação (subitens registros do sistema de alerta de inundação por área alvo e listagem de alertas de inundação emitidos desde que o sistema de alerta de inundação entrou em operação) foram encontrados apenas em modo fechado (PDF e Mapa Interativo).

\section{Vulnerabilidade}

A dimensão de vulnerabilidade é formada a partir de sete itens principais, que se referem às curvas de vulnerabilidade para: ativos agregados se referem às funções para calcular o dano econômico em todos os ativos em uma determinada área do nível de perigo; tipo de cobertura territorial tratam das funções para calcular o dano econômico do nível de e tipo de cobertura do solo; infraestrutura crítica se referem às funções para calcular os danos em infraestruturas críticas do nível de perigo; população servem para calcular o número de pessoas afetadas e fatalidades do nível de perigo e densidade populacional; atividades econômicas tratam das funções para calcular o dano econômico do nível de risco e informações sobre atividades econômicas; edifícios servem para calcular o dano econômico em edifícios a partir do nível de risco e tipo de construção; e atividades agrícolas se referem às funções para calcular o dano econômico na agricultura de nível de risco e produção agrícola.

Nenhuma informação foi encontrada referente a estes itens. Nem em dados abertos e nem em dados fechados ou restritos. Esta foi a única dimensão que zerou.

\section{Risco}

A dimensão de risco é formada pelo item indicadores de risco de estudos anteriores. Este item possui em sua estrutura sete subitens, que dentre sua composição, 29\% apresentaram dados abertos. Mapas de risco quadriculados (mosaico de imagens) e Mapas de risco agregados por bairro, foram os únicos onde foi possível encontrar dados aberto em formato .SHP. Para os demais subitens, só foram encontrados arquivos fechados em formato PDF: nível de risco com indicação de prejuízos econômicos e danos humanos/materiais; nível de risco com indicação de perdas de um evento específico; nível de risco com indicação de perdas para um determinado período de retorno; risco tabelado por uma determinada área, por exemplo, curva de excedência de perdas, tabelas de perdas de eventos, tabelas de perdas de anos ou dados históricos de perda; e ativos expostos para um determinado evento (histórico ou associado a um período de retorno).

$\mathrm{O}$ resumo dos itens e formatos de dados encontrados pode ser observado no Quadro 3. 
Quadro 3. Resumo das dimensões, itens e formatos encontrados (fonte: elaborado pelos autores)

\begin{tabular}{|c|c|c|c|}
\hline \multicolumn{4}{|c|}{ Exposição } \\
\hline Itens & Formato & Itens & Formato \\
\hline Zoneamento & $\begin{array}{l}\text { Aberto: SBN; SBX; DWG; } \\
\text { DBF; ZIP } \\
\text { Fechado: JPEG, PDF }\end{array}$ & $\begin{array}{l}\text { Uso e Ocupação } \\
\text { do Solo }\end{array}$ & $\begin{array}{l}\text { Aberto: não identificado } \\
\text { Fechado: JPEG, PDF }\end{array}$ \\
\hline Cobertura da Terra & $\begin{array}{l}\text { Aberto: CSV; JSON; ZIP; } \\
\text { KML; HTML } \\
\text { Fechado: Jpeg, PDF }\end{array}$ & Loteamentos & $\begin{array}{l}\text { Aberto: SBN; SBX; DWG; } \\
\text { DBF; CSV; ZIP } \\
\text { Fechado: JPEG, PDF }\end{array}$ \\
\hline População & $\begin{array}{l}\text { Aberto: DWG; CSV } \\
\text { Fechado: JPEG; PDF }\end{array}$ & Domicílio & $\begin{array}{l}\text { Aberto: DWG; CSV } \\
\text { Fechado: JPEG; PDF }\end{array}$ \\
\hline $\begin{array}{l}\text { Atividades } \\
\text { econômicas }\end{array}$ & $\begin{array}{l}\text { Aberto: CSV } \\
\text { Fechado: JPEG; PDF }\end{array}$ & $\begin{array}{l}\text { Registro de } \\
\text { empresas }\end{array}$ & $\begin{array}{l}\text { Aberto: CSV } \\
\text { Fechado: Lista Interativa }\end{array}$ \\
\hline $\begin{array}{l}\text { Edifícios e ativos } \\
\text { tecnológicos }\end{array}$ & $\begin{array}{l}\text { Aberto: CSV } \\
\text { Fechado: JPEG; PDF }\end{array}$ & $\begin{array}{l}\text { Equipamentos } \\
\text { Municipais }\end{array}$ & $\begin{array}{l}\text { Aberto: CSV } \\
\text { Fechado: JPEG; PDF }\end{array}$ \\
\hline $\begin{array}{l}\text { Infraestrutura } \\
\text { crítica }\end{array}$ & $\begin{array}{l}\text { Aberto: SBN; SBX; DWG; } \\
\text { DBF; CSV; SVG; ZIP } \\
\text { Fechado: PDF; JPEG; PNG; } \\
\text { GIF; Tabela e Mapas } \\
\text { Interativos }\end{array}$ & $\begin{array}{l}\text { Patrimônio } \\
\text { histórico e cultural }\end{array}$ & $\begin{array}{l}\text { Aberto: CSV } \\
\text { Fechado: PDF }\end{array}$ \\
\hline $\begin{array}{l}\text { Infraestrutura } \\
\text { administrativa }\end{array}$ & $\begin{array}{l}\text { Aberto: CSV } \\
\text { Fechado: PDF }\end{array}$ & $\begin{array}{l}\text { Abastecimento } \\
\text { alimentar }\end{array}$ & $\begin{array}{l}\text { Aberto: CSV } \\
\text { Fechado: JPEG; PDF }\end{array}$ \\
\hline $\begin{array}{l}\text { Infraestrutura } \\
\text { turística }\end{array}$ & $\begin{array}{l}\text { Aberto: CSV } \\
\text { Fechado: JPEG; PDF }\end{array}$ & $\begin{array}{l}\text { Equipamentos } \\
\text { esportivos }\end{array}$ & $\begin{array}{l}\text { Aberto: SBN; SBX; DWG; } \\
\text { DBF; CSV; ZIP } \\
\text { Fechado: JPEG, PDF }\end{array}$ \\
\hline $\begin{array}{l}\text { Equipamentos } \\
\text { religiosos }\end{array}$ & $\begin{array}{l}\text { Aberto: CSV } \\
\text { Fechado: JPEG; PDF }\end{array}$ & $\begin{array}{l}\text { Áreas verdes e de } \\
\text { lazer }\end{array}$ & $\begin{array}{l}\text { Aberto: SBN; SBX; DWG; } \\
\text { DBF; CSV; ZIP } \\
\text { Fechado: JPEG, PDF }\end{array}$ \\
\hline Áreas de risco & $\begin{array}{l}\text { Aberto: CSV } \\
\text { Fechado: PDF }\end{array}$ & Áreas irregulares & $\begin{array}{l}\text { Aberto: SBN; SBX; DWG; } \\
\text { DBF; CSV; ZIP } \\
\text { Fechado: JPEG, PDF }\end{array}$ \\
\hline Vazios Urbanos & $\begin{array}{l}\text { Aberto: SBN; SBX; DWG; } \\
\text { DBF; CSV; ZIP } \\
\text { Fechado: JPEG, PDF }\end{array}$ & $\begin{array}{l}\text { Serviços de } \\
\text { emergência }\end{array}$ & $\begin{array}{l}\text { Aberto: CSV } \\
\text { Fechado: JPEG; PDF }\end{array}$ \\
\hline Segurança & $\begin{array}{l}\text { Aberto: XLSX; CSV } \\
\text { Fechado: não identificado }\end{array}$ & & \\
\hline
\end{tabular}

\begin{tabular}{l|l|l|l}
\hline \multicolumn{4}{c|}{ Dados de base } \\
\hline \multicolumn{1}{c|}{ Itens } & \multicolumn{1}{c|}{ Formato } & \multicolumn{1}{c}{ Itens } & \multicolumn{1}{c}{ Formato } \\
\hline $\begin{array}{l}\text { Limites } \\
\text { administrativos }\end{array}$ & $\begin{array}{l}\text { Aberto: SBN; SBX; DWG; } \\
\text { DBF; ZIP } \\
\text { Fechado: JPEG; PDF; Mapa } \\
\text { Interativo }\end{array}$ & Imagens aéreas & $\begin{array}{l}\text { Aberto: SBN; SBX; DWG; } \\
\text { DBF; ZIP } \\
\text { Fechado: não identificado }\end{array}$ \\
\hline $\begin{array}{l}\text { Modelo de } \\
\text { elevação digital }\end{array}$ & $\begin{array}{l}\text { Aberto: CSV, JSON, ZIP, } \\
\text { KML, HTML } \\
\text { Fechado: não identificado }\end{array}$ & Tipo de solo & $\begin{array}{l}\text { Aberto: CSV; JSON; ZIP; } \\
\text { KML; HTML } \\
\text { Fechado: PDF }\end{array}$ \\
\hline $\begin{array}{l}\text { Topografia e } \\
\text { orientação }\end{array}$ & $\begin{array}{l}\text { Aberto: não identificado } \\
\text { Fechado: Mapa Interativo }\end{array}$ & Altimetria & $\begin{array}{l}\text { Aberto: SBN; SBX; DWG; } \\
\text { DBF; ZIP } \\
\text { Fechado: PDF }\end{array}$ \\
\hline Hipsometria & $\begin{array}{l}\text { Aberto: não identificado } \\
\text { Fechado: PDF }\end{array}$ & Declividade & $\begin{array}{l}\text { Aberto: não identificado } \\
\text { Fechado: PDF }\end{array}$ \\
\hline Cobertura vegetal & $\begin{array}{l}\text { Aberto: SBN; SBX; DWG; } \\
\text { DBF; ZIP }\end{array}$ & Corpos d'água & $\begin{array}{l}\text { Aberto: SBN; SBX; DWG; } \\
\text { DBF; ZIP } \\
\text { Fechado: não identificado }\end{array}$ \\
\hline $\begin{array}{l}\text { Limites de bacias } \\
\text { hidrográficas }\end{array}$ & Aberto: ZIP & Rede hidrográfica & $\begin{array}{l}\text { Aberto: SBN; SBX; DWG; } \\
\text { DBF; ZIP } \\
\text { Fechado: PDF }\end{array}$ \\
\hline
\end{tabular}




\begin{tabular}{|c|c|c|c|}
\hline $\begin{array}{l}\text { Mapa } \\
\text { Geomorfológico }\end{array}$ & $\begin{array}{l}\text { Aberto: CSV, JSON, ZIP, } \\
\text { KML, HTML } \\
\text { Fechado: }\end{array}$ & Arruamento & $\begin{array}{l}\text { Aberto: SBN; SBX; DWG; } \\
\text { DBF; ZIP } \\
\text { Fechado: não identificado }\end{array}$ \\
\hline Área urbanizada & $\begin{array}{l}\text { Aberto: não identificado } \\
\text { Fechado: PDF }\end{array}$ & $\begin{array}{l}\text { Áreas } \\
\text { impermeáveis }\end{array}$ & $\begin{array}{l}\text { Aberto: não identificado } \\
\text { Fechado: PDF }\end{array}$ \\
\hline Pavimentação & $\begin{array}{l}\text { Aberto: CSV } \\
\text { Fechado: PDF; JPEG }\end{array}$ & & \\
\hline \multicolumn{4}{|c|}{ Perigo } \\
\hline Itens & Formato & Itens & Formato \\
\hline $\begin{array}{l}\text { Mapas de risco de } \\
\text { inundação }\end{array}$ & $\begin{array}{l}\text { Aberto: não identificado } \\
\text { Fechado: JPEG; PDF }\end{array}$ & Áreas Inundáveis & $\begin{array}{l}\text { Aberto: não identificado } \\
\text { Fechado: JPEG; PDF }\end{array}$ \\
\hline $\begin{array}{l}\text { Cotas de } \\
\text { Inundação }\end{array}$ & $\begin{array}{l}\text { Aberto: não identificado } \\
\text { Fechado: não identificado }\end{array}$ & $\begin{array}{l}\text { Dados } \\
\text { meteorológicos }\end{array}$ & $\begin{array}{l}\text { Aberto: XLS; TXT } \\
\text { Fechado: JPEG; PNG; PDF; } \\
\text { Mapa Interativo }\end{array}$ \\
\hline $\begin{array}{l}\text { Dados } \\
\text { hidrológicos }\end{array}$ & $\begin{array}{l}\text { Aberto: XLS; TXT } \\
\text { Fechado: PDF }\end{array}$ & $\begin{array}{l}\text { Medidas de } \\
\text { proteção contra } \\
\text { inundações }\end{array}$ & $\begin{array}{l}\text { Aberto: XLS; TXT } \\
\text { Fechado: PDF }\end{array}$ \\
\hline $\begin{array}{l}\text { Registros } \\
\text { históricos de } \\
\text { eventos de risco }\end{array}$ & $\begin{array}{l}\text { Aberto: não identificado } \\
\text { Fechado: PDF }\end{array}$ & $\begin{array}{l}\text { Avisos/alertas de } \\
\text { inundação }\end{array}$ & $\begin{array}{l}\text { Aberto: CSV } \\
\text { Fechado: PDF }\end{array}$ \\
\hline \multicolumn{4}{|c|}{ Vulnerabilidade } \\
\hline \multicolumn{3}{|c|}{ Itens } & Formato \\
\hline \multicolumn{3}{|c|}{ Curvas de vulnerabilidade para ativos agregados } & \multirow{7}{*}{$\begin{array}{l}\text { Aberto: não identificado } \\
\text { Fechado: não identificado }\end{array}$} \\
\hline \multicolumn{3}{|c|}{ Curvas de vulnerabilidade para infraestrutura crítica } & \\
\hline \multicolumn{3}{|c|}{ Curvas de vulnerabilidade para atividades econômicas } & \\
\hline \multicolumn{3}{|c|}{ Curvas de vulnerabilidade para atividades agrícolas } & \\
\hline \multicolumn{3}{|c|}{ Curvas de vulnerabilidade por tipo de cobertura territorial } & \\
\hline \multicolumn{3}{|c|}{ Curvas de vulnerabilidade para população } & \\
\hline \multicolumn{3}{|c|}{ Curvas de vulnerabilidade para edifícios } & \\
\hline \multicolumn{4}{|c|}{ Risco } \\
\hline & Itens & & Formato \\
\hline \multicolumn{2}{|c|}{ Indicadores de risco de estudos anteriores } & $\begin{array}{l}\text { Aberto: ZIP } \\
\text { Fechado: PDF }\end{array}$ & \\
\hline
\end{tabular}

$\mathrm{O}$ resumo dos indicadores de abertura de dados alcançados por dimensão pode ser observado na Figura 1.

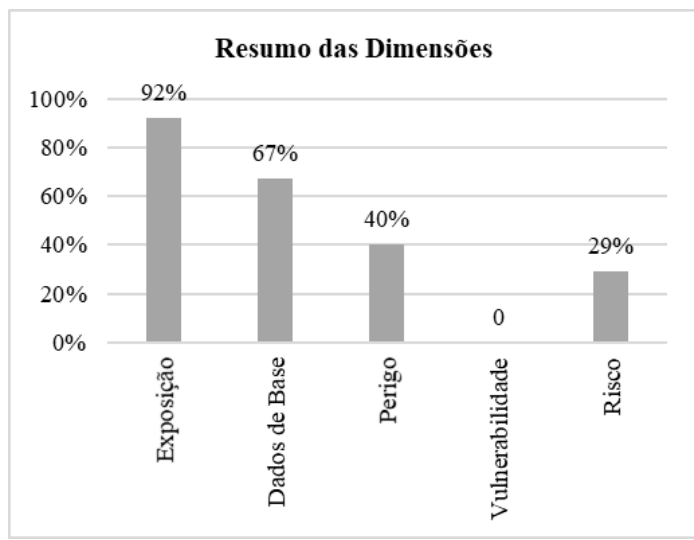

Figura 1. Resumo dos resultados por dimensão (fonte: elaborada pelos autores).
De maneira geral, $51 \%$ dos itens investigados apresentaram arquivos abertos, enquanto $36 \%$ apenas apresentaram arquivos fechados e 13\% ausência total de informações. Foram encontrados dados abertos tabulares, geoespaciais e em outros formatos, tais quais: .CSV, .JSON, .DBF, .SHP, .SBN, .SVG, .KML, .HTML, .SBX, e .DWG. Os sites que continham o maior volume de informação acumulado foram "Curitiba em Dados" e "IPPUC".

É possível observar que metade dos dados pesquisados não foram encontrados em formato aberto. Em parte, justifica-se que os sistemas públicos não possuem padronização nem das plataformas e nem das bases de dados constituintes (Silva \& Galvão 2018).

Não obstante, dentre as dimensões analisadas, observa-se que a maior parte dos 
dados abertos se concentra na Exposição, principalmente quanto às informações de infraestrutura, áreas verdes, áreas irregulares, cobertura do solo e zoneamento. Este perfil pode ser explicado pela tipologia dos dados, tendo em vista que são informações abrangentes, e que por consequência, mais setores fazem uso delas.

Partindo deste princípio, observa-se que na dimensão da Vulnerabilidade não foi possível encontrar nenhum dado. Como se trata de informações específicas, tanto em relação à área que pertencem, quanto ao tipo de arquivo buscado, as chances de os mesmos estarem disponíveis reduzem significativamente. Infelizmente, a presente dimensão, em conjunto às de Perigo e Riscos, são as que buscam por dados críticos para a resiliência, especificamente sobre as inundações, e como pôde ser observado, também são àquelas com menos informações encontradas.

Destaca-se também que, embora nem todos os itens pesquisados possuam dados abertos disponíveis, a existência de arquivos fechados é um indicativo de que as informações podem ser encontradas. Se a fonte do arquivo for identificada, será possível solicitar diretamente ao órgão responsável pelo formato aberto.

No entanto, afirma-se que a necessidade de compartilhamento de dados aberto é iminente, sendo ideal o incentivo contínuo pela disponibilização dos mesmos. Quanto mais dados e maior for a diversidade disponível, maior será a parcela da sociedade beneficiada, tendo em vista que essas informações são essenciais para a produção de novas pesquisas e desenvolvimento de projetos que visem a redução de riscos e a resiliência nas cidades.

\section{Resultados dos questionários}

A partir deste cenário, foi realizada a aplicação de questionários sobre o acesso à informação e conhecimento sobre dados abertos. Ao total, 30 questionários foram respondidos por cidadãos de Curitiba, sendo metade dos agentes sociais do gênero masculino e a outra metade feminino. A determinação da amostra se deu pela diversidade de experiência profissional (estudantes, autônomos, empregados dos setores industrial, comercial e serviço) e também pela faixa etária. A idade variou entre 19 e 54 anos, com a maior parcela pertencente ao grupo de até 30 anos (60\%). Em relação a formação, $30 \%$ possuem ensino superior completo, $27 \%$ nível de pósgraduação ou acima, $27 \%$ estão cursando o ensino superior, $13 \%$ possuem ensino médio e $3 \%$ ensino fundamental.

Após a caracterização, foram realizadas cinco perguntas sobre o acesso à informação de riscos de desastres no município. Metade dos entrevistados afirmam já ter recebido algum tipo de informação sobre o tema, enquanto a outra metade reconhece que as informações chegam apenas após o desastre já ter ocorrido. Dentre os meios de comunicação por onde as informações chegam, a televisão, as redes sociais e os sites foram os mais citados, seguidos por SMS e rádio (Figura 2). Destaca-se que todas as pessoas que recebem os alertas da Proteção e Defesa Civil por SMS, afirmaram anteriormente já ter recebido algum tipo de informação sobre o risco de desastres em Curitiba.

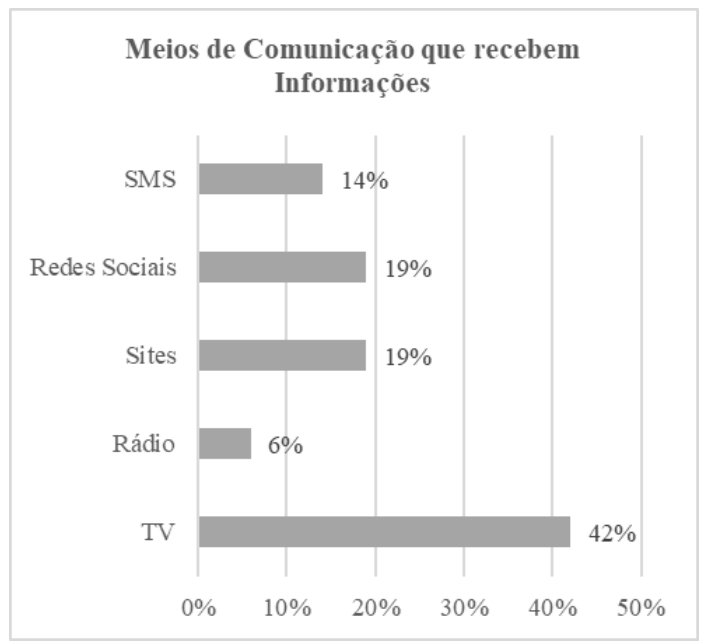

Figura 2. Meios de comunicação que recebem informações (fonte: elaborada pelos autores).

Quando questionados se já precisaram buscar por alguma informação sobre riscos ou sobre algum desastre específico nos sites oficiais do município, apenas $20 \%$ respondeu que sim. Destes metade conseguiu encontrar o que procurava, mas todos afirmaram que foi difícil procurar os dados nas páginas disponíveis. Embora o percentual de busca tenha sido baixo, todos os agentes afirmaram considerar importante que as informações sobre riscos devem ser divulgadas para a 
população, pois instruem a população quanto aos devidos cuidados que devem ser tomados diante de qualquer tipo de incidente ou desastre natural, com antecedência.

A partir do conhecimento sobre as informações que são recebidas pela população, os entrevistados foram abordados acerca de seu conhecimento sobre Dados Abertos. A maioria nunca tinha ouvido falar neste termo $(56,7 \%)$. No entanto, os entrevistados possuem conhecimento sobre a disponibilidade de arquivos .CVS, por exemplo, que podem baixar em alguns sites. Embora a maior parte não tenha apresentado o conhecimento pelo termo, todos concordam que a disponibilidade deles é importante para que a população tenha a oportunidade no desenvolvimento de trabalhos e pesquisas.

Em relação ao acesso a estes dados, $90 \%$ dos agentes afirma desconhecer se existe algum portal da prefeitura próprio para disponibilização destes dados. Destaca-se que alguns deles citaram conhecer apenas o site do Instituto Brasileiro de Geografia e Estatística (IBGE) para este fim. Sobre confiança perante a disponibilização por parte do governo, as pessoas foram questionadas se acreditam que podem encontrar informações diversas, de todas as áreas setoriais pertencentes a prefeitura no formato aberto. $87 \%$ não acredita que haja acesso para todos os dados.

Por fim, tendo em vista que a prefeitura municipal de Curitiba disponibilizou o Portal de Dados Abertos após a Lei de Acesso à Informação, buscou-se identificar se o governo utilizou algum mecanismo para incentivar a população quanto ao uso dos dados disponibilizados. Para isto, foi perguntado aos agentes se eles receberam qualquer incentivo por parte dos gestores do município, e 93\% responderam que não.

Após a identificação deste cenário, também pôde-se realizar algumas análises a partir de grupos amostrais apresentados na caracterização dos entrevistados. Em relação ao gênero, as mulheres apresentaram ter maior identificação pelas informações recebidas sobre os riscos de desastres no município. Foram $67 \%$ contra 33\% dos homens. Os meios de comunicação mais utilizados são a Tv e a Internet (redes sociais e sites), onde as mulheres também se sobressaem em relação aos homens quanto a utilização (73\% para TV e 64\% para internet). Outro quesito identificado é que apenas as mulheres citaram a utilização de rádio. Quanto aos dados abertos, $53 \%$ das mulheres já tinham conhecimento prévio sobre a existência dos mesmos, enquanto dos homens, apenas $27 \%$ tinham ouvido falar.

Nos grupos de faixa etária, metade das pessoas com até 30 anos e $44 \%$ dos acima dos 30, afirmam ter recebido alguma informação sobre riscos. Foi identificada uma inversão na utilização de meios de comunicação. Os agentes até 30 anos usam primeiro as redes sociais e sites e depois a $\mathrm{Tv}$, enquanto os acima de 30 usam primeiramente a Tv e depois a internet. Outro ponto identificado, é que $80 \%$ das pessoas que possuem o sistema de alerta por SMS pertencem ao grupo de até 30 anos. O mesmo contraste aparece no conhecimento sobre dados abertos, onde 53\% dos com até 30 anos afirmam já ter ouvido falar, contra apenas $29 \%$ do acima de 30 anos.

Considerando a escolarização dos agentes sociais, $63 \%$ dos que possuem nível superior completo e acima, afirmam já ter recebido algum tipo de informação sobre os riscos de desastres que podem ocorrer em Curitiba. Os meios de comunicação citados são TV $(40 \%)$, sites (20\%) e SMS (20\%), redes sociais $(16 \%)$ e rádio (4\%). Quando questionados se já ouviram falar sobre dados abertos anteriormente, $63 \%$ responderam que sim e $37 \%$ que não.

Em contrapartida, aqueles que possuem nível fundamental, médio ou superior incompleto, e que afirmam ter recebido algum tipo de informação, representam apenas $29 \%$. Os meios de comunicação mais utilizados são televisão e redes sociais ( $46 \%$ e $27 \%$, respectivamente), seguidos de sites (18\%) e rádio $(9 \%)$. Observa-se que nenhum dos agentes nestes níveis de escolarização recebe informações via SMS, inferindo que não possuem o cadastro de alerta a desastres da Proteção e Defesa Civil de Curitiba. Em relação ao conhecimento anterior sobre dados abertos, apenas $14 \%$ responderam que sim enquanto $86 \%$ que não. 


\section{Considerações finais}

Com o desenvolvimento desta pesquisa, foi possível atender o objetivo central do artigo, respondendo às duas questões-problema. No primeiro momento, buscou-se analisar se os dados abertos estão sendo disponibilizados e são suficientes para serem utilizados como instrumento de resiliência às inundações. Embora tenham sido encontrados dados abertos da parte que caracteriza o município, são poucos os referentes a temática de riscos de inundação. Destaca-se que algumas informações se apresentam com registros muito antigos, inferindo-se que não há atualização dos dados disponíveis. A maioria dos arquivos disponibilizados são fechados, em formato PDF e JPEG.

Cabe aqui destacar que, dependendo do tipo de dado aberto, faz-se necessário disponibilizar uma visualização prévia, um arquivo fechado (imagem ou PDF) ou até mesmo interativo no próprio site para que seja possível observar que informações será possível acessar. Exemplos disto são o .SHP ou .DWG, padrões da indústria, tanto em Planejamento Urbano e Territorial, quanto nas áreas correlatas, onde muitas pessoas não têm acesso aos tipos de programa que os abrem. A dificuldade de visualização prévia é um problema neste caso. Pois ela normalmente ocorre em geoportais que necessitam de investimentos altos, ou em um formato imagem, e dessa maneira não possibilitam a realização de alterações e melhorias.

Outro ponto importante se refere às plataformas de acesso a estes dados. Não existe uma plataforma única com todos os arquivos. O Portal de Dados Abertos não possui variedade de informação. Os dados disponíveis estão em maior parte em arquivo para planilhamento, não possuindo arquivos georreferenciados para elaboração de mapas. Desta forma foi necessário buscá-los em diversos sites, pois o acesso é difícil, nem mesmo utilizando os mecanismos de busca na internet com palavras-chave.

Após esta identificação, buscou-se compreender a importância dos dados abertos e sua influência para a promoção da resiliência. É por meio da disponibilização de dados que a população pode ter mais acesso à informação, e consequentemente, que entendam as características do meio em que vivem. Por meio desse processo, as atitudes e ações das pessoas podem mudar para melhor. Por conseguinte, os grupos vulneráveis terão a capacidade de enfrentar o risco, bem como implementar medidas preventivas para reduzir a vulnerabilidade e o potencial de danos, como as obras civis de prevenção à desastres. $\mathrm{O}$ fornecimento de dados não se traduz em preparação. É necessário compreender quais dados são relevantes e úteis para diferentes grupos populacionais em distintas áreas. Bem como avaliar o seu recebimento a partir das experiências construídas através de interações na sociedade.

Deste modo, foi realizada a aplicação dos questionários, a fim de analisar as relações entre os usuários e a disponibilização de dados. Logo, observou-se que embora o site de dados abertos de Curitiba tenha sido lançado após a lei de acesso à informação, os participantes não foram informados sobre este assunto em nenhum momento por parte dos gestores. Os participantes também afirmam não receberem nenhum incentivo por parte do governo municipal para a utilização de dados abertos, sendo importante à gestão se atentar quanto a efetividade dos mecanismos adotados para este fim atualmente.

Por fim, um dos maiores problemas encontrados é que nenhum dos sites onde as buscas foram realizadas se refere a plataforma oficial de gerenciamento de riscos e desastres e informações correlatas, mesmo sendo um tema emergentes e vem causando tantos impactos negativos nas cidades. Neste momento, se faz necessária a iniciativa governamental em buscar por medidas que impulsionem o sistema de dados abertos referentes a gestão de riscos e desastres dos municípios, superando as dificuldades de implementar estruturas para a interoperabilidade das informações produzidas por setores diferentes. Além disso, é importante que os demais setores da sociedade (privado, não-governamental e universidades), busquem por critérios que possibilitem a abertura dos dados disponíveis e os compartilhem com a população.

Conclui-se que os dados abertos são muito importantes para a promoção da resiliência, 
influenciando diretamente na formulação de estratégias para a redução dos riscos nas cidades. Como foi observado, compartilhar diferentes formatos de arquivos faz com que vários setores da sociedade sejam alcançados, em especial às universidades, que apresentam o desenvolvimento contínuo de trabalhos e pesquisas para a busca de soluções dos problemas urbanos. Quanto mais dados estiverem disponíveis, maiores serão os instrumentos para o aperfeiçoamento de ferramentas existentes, o combate da vulnerabilidade social e a redução e

\section{Referências}

Beck, U. (1992) Risk Society: Towards a New Modernity. Londres, Sage.

BI-CEPDEC. (2020) Relatório de Desastres do Paraná. Curitiba: Coordenadoria Estadual de Proteção e Defesa Civil do Paraná.

Calkins, J. (2015) Moving Forward after Sendai: How Countries Want to Use Science, Evidence and Technology for Disaster Risk Reduction. PLOS Currents Disasters, 1-10 Disponível em: https://doi.org/10.1371/currents.dis.22247d6293d4 109d09794890bcda1878

Carvalho, L., Carrasco, Ú., Farinha, M., Batista, S., Fernandes, J. M., Sousa, G. \& Leitão, N. (2013) Risco, desastre e resiliência: um desafio para a cidade da Amadora. Em: Anais do Congresso da Geografia Portuguesa. Universidade de Évora, Portugal. Disponível em: https://bit.ly/3cBM4Xe. [Consultado em: 16 de novembro de 2019].

Christensen, E. V., Lewis, D. \& Armesto, M. F. (org.) (2016) Guía de Resiliencia Urbana 2016. México, EUM. SEGOB. SEDATU. SNPC. ONU.

Coordenação da Região Metropolitana de Curitiba. (2006) Plano de desenvolvimento integrado da Região Metropolitana de Curitiba: propostas de ordenamento territorial e novo arranjo institucional. Curitiba, COMEC.

Coordenadoria Estadual de Proteção e Defesa Civil. (2019) Ocorrências de desastres no Estado do Paraná entre 1980 e 2018. Curitiba, CEPDEC.

Corrêa, A. S., Corrêa, P. L. P. \& Silva, F.S.C. (2014) Transparency Portals Versus Open Government Data. An Assessment of Openness in Brazilian Municipalities. Anais da 15th Annual International Conference on Digital Government Research. Aguascalientes, 178-185. Disponível em: https://doi.org/10.1145/2612733.2612760

Farias, J. A. (2017) Resiliência: um bom conceito para o projeto e a reforma urbana? Anais do XVII Encontro Nacional ANPUR. São Paulo. Disponível mitigação de áreas suscetíveis a riscos. Deste modo, a integração multisetorial e o fortalecimento das cidades ocorrem de maneira abrangente por meio da resiliência a eventos extremos.

\section{Agradecimentos}

O presente trabalho foi realizado com apoio da Coordenação de Aperfeiçoamento de Pessoal de Nível Superior - Brasil (CAPES) - Código de Financiamento 001.

em: https://bit.ly/2XgP1Gj. [Consultado em: 16 de novembro de 2019].

Folke, C., Carpenter, S., Elmqvist, T., Gunderson, L., Holling, C. S. \& Walker, B. (2002) Resilience and sustainable development: building adaptive capacity in a world of transformations. Ambio, 31(5), 437-440. Disponível em: https://doi.org/10.1579/0044-7447-31.5.437

Freitas, C. M. De, Silva, D. R. X., Sena, A. R. M. de, Silva, E. L., Sales, L. B. F., Carvalho, M. L. De, Mazoto, M. L., Barcellos, C., Costa, A. M., Oliveira, M. L. C. \& Corvalán, C. (2014). Desastres naturais e saúde: uma análise da situação do Brasil. Ciência \& Saúde Coletiva, 19(9), 3645-3656. Disponível em: https://doi.org/10.1590/141381232014199.00732014

Geissler, H.J. \& Loch, R.E.M. (2004) Análise Histórica das enchentes em Curitiba - PR, medidas propostas e consequências observadas. Florianópolis, Universidade Federal de Santa Catarina.

Gil, C. A. (2019) Métodos e Técnicas de Pesquisa Social. São Paulo: Atlas.

Goëta, S. (2012) Open data: qu'ouvre-t-on avec des données publiques? Mémoire de master. Paris, Université Paris Sorbonne.

Hogan, D. J. \& Marandola Jr., E. (Orgs.) (2009) População e mudança climática: dimensões humanas das mudanças ambientais globais. Campinas, Universidade Estadual de Campinas.

Hosseini, S., Barker, K. \& Ramirez-Marquez, J. E. (2015) A review of definitions and measures of system resilience. Reliability Engineering and System Safety, 145, 47-61. Disponível em: https://doi.org/10.1016/j.ress.2015.08.006

Instituto de Pesquisa e Planejamento Urbano de Curitiba. (2020) Curitiba em Dados. Curitiba: IPPUC. Disponível em: https://bit.ly/3cGOhQY. [Consultado em: 25 de feveveiro de 2020].

Instituto de Pesquisa e Planejamento Urbano de Curitiba. (2020) Dados Geográficos. Curitiba: 
IPPUC. Disponível em: https://bit.ly/3fYrORt. [Consultado em: 20 de fevereiro de 2020].

Janssen, M., Charalabidis, Y., \& Zuiderwijk, A. (2012) Benefits, adoption barriers and myths of open data and open government. Information Systems Management, 29(4), 258-268. Disponível em:

https://doi.org/10.1080/10580530.2012.716740

Johnson, P. A., Corbett, J. M., Gore, C., Robinson, P., Allen, P., \& Sieber, R. (2015) A Web of Expectations: Evolving relationships in community participatory Geoweb projects. ACME, 14(3), 827848. Disponível em: https://bit.ly/3bFrBPT. [Consultado em: 12 de novembro de 2019].

Kassen, M. (2013) A promising phenomenon of open data: A case study of the Chicago open data project. Government Information Quarterly, 30(4), 508-513. Disponível em: https://doi.org/10.1016/j.giq.2013.05.012

Landry, J.-N., Webster, K., Wylie, B. \& Robinson, P. (2016) How Can We Improve Urban Resilience with Open Data?: London, Open Data Institute.

Leichenko, R. (2011) Climate change and urban resilience. Current opinion in environmental sustainability, 3(3), 164-168. Disponível em: https://doi.org/10.1016/j.cosust.2010.12.014

Li, G., Zhao, J., Murray, V., Song, C. \& Lianchong, Z. (2019) Gap analysis on open data interconnectivity for disaster risk research. Geospatial Information Science, 22(1), 45-58. Disponível em: https://doi.org/10.1080/10095020.2018.1560056

Mehmood, A. (2015) Of resilient places: planning for urban resilience. European Planning Studies, 24(2), 407-419. Disponível em: https://doi.org/10.1080/09654313.2015.1082980

Mendonça, F., Deschamps, M. \& Lima, M. D. V. (2013) A cidade e as mudanças globais: (intensificação?) riscos e vulnerabilidades socioambientais na RMC - Região Metropolitana de Curitiba/PR. Em: Ojima, R. \& Marandola Junior, E. (org.). Mudanças climáticas e as cidades: novos e antigos debates na busca da sustentabilidade urbana e social. São Paulo, Blucher, pp.129-162.

Murnane, R., S., Loughlin, T., Rossetto, V., Silva, A., Ali, C. \& Galasso, C. (2019) Extensible Data Schema for Multiple Hazards, Exposure and Vulnerability. GAR19 Contributing Paper, UNISDR. Disponível em: https://bit.ly/2Z6v4V7. [Consultado em: 10 de novembro de 2019].

OpenDRI. (2019) Open Data for Resilience Index. Documentation. Disponível em: https://bit.ly/3bE38dV. [Consultado em: 20 de fevereiro de 2020].
Prefeitura Municipal de Curitiba. (2020) Dados Abertos. Curitiba: PMC. Disponível em: https://bit.ly/2y790hR. [Consultado em: $28 \mathrm{de}$ fevereiro de 2020].

Robinson, P. J. \& Johnson, P. A. (2016) Civic hackathons: New terrain for local governmentcitizen interaction? Urban Planning, 1(2), 65-74. Disponível http://dx.doi.org/10.17645/up.v1i2.627

Santana, R. C. G. \& Rodrigues, F. de A. (2013) Acessando dados para visualização de afinidades nas votações entre parlamentares do Senado. Informação \& Sociedade, 23(1), 49-59. Disponível em: https://bit.ly/2LyI9OP. [Consultado em: 18 de novembro de 2019].

Serwadda, D., Ndebele, P., Grabowski, M. K., Bajunirwe, F. \& Wanyenze, R. K. (2018) Open data 27 sharing and the Global South-Who benefits? Science, (359), 642-643. Disponível em: https://doi.org/10.1126/science.aap8395

Sharifi, A. (2019) Resilient urban forms: A macroscale analysis. Cities, 85, 1-14. Disponível em: https://doi.org/10.1016/j.cities.2018.11.023

Sharifi, A., Chelleri, L., Fox-Lent, C., Grafakos, S., Pathak, M., Olazabal, M. \& Yamagata, Y. (2017) Conceptualizing dimensions and characteristics of urban resilience: Insights from a co-design process. Sustainability, 9(6), 1032. Disponível em: https://doi.org/10.3390/su9061032

Sharifi, A. \& Yamagata, Y. (2016) Principles and criteria for assessing urban energy resilience: A literature review. Renewable and Sustainable Energy Reviews, 60, 1654-1677. Disponível em: https://doi.org/10.1016/j.rser.2016.03.028

Silva, A. R. I. \& Galvão, M. C. (2018) A importância do uso de dados abertos pelo poder público para o fortalecimento da governança pública. Anais do IX Seminário de Ensino, Pesquisa e Extensão. Universidade Federal do Rio Grande do Norte, Natal. Disponível em: https://bit.ly/3fSuC2x. [Consultado em: 22 de janeiro de 2020].

Sulaiman, S. N. \& Aledo, A. (2016) Desastres naturais: convivência com o risco. Estudos Avançados: São Paulo, 30(88). Disponível em: https://doi.org/10.1590/s0103-

40142016.30880003

Tyler, S. \& Moench, M. (2012) A framework for urban climate resilience. Climate and Development, 4(4), 311-326. Disponível em: https://doi.org/10.1080/17565529.2012.745389

UNDRR. (2019) Making Cities Resilient: My city is getting ready. Geneva, United Nations Office for Disaster Risk Reduction. Disponível em: 
https://bit.ly/2WzQw2W. [Consultado em: 08 de novembro de 2019].

UNDRR. (2019) Global Assessment Report on Disaster Risk Reduction. Geneva, United Nations Office for Disaster Risk Reduction. Disponível em: https://bit.ly/2Tby7r0. [Consultado em: $10 \mathrm{de}$ novembro de 2019].

Veyret, Y. \& Richemond, N. M. (2007) Os tipos de riscos. Em: Veyret, Y. Os riscos: O homem como agressor e vítima do meio ambiente. São Paulo, Contexto, pp.63-76.

Zanella, M. E. (2006) Inundações urbanas em Curitiba/PR: impactos, riscos e vulnerabilidade socioambiental no bairro Cajuru. Tese de Doutoramento não publicada. Universidade Federal do Paraná, Curitiba.

\title{
Tradução do título, resumo e palavras-chave
}

\author{
Availability of open data for flood resilience in Curitiba (Paraná)
}

Abstract. Urban managers face the challenge of lack of updated data. The Information Access Law emphasized the importance of making data openly accessible, so that the population can use it freely. At the same time, the incidence of disasters in cities has increased in recent years, with the production of disaster risk data being one of the government's functions. The more detailed the identification of the risks associated with natural hazards, the more effective the measures for reducing impacts will become. The objective of this work is to understand whether open data are being made available and are sufficient to be used as an instrument of resilience to floods. To this end, the application was carried out in the city of Curitiba, in three stages: (1) searching for files on the city hall's open data portal; (2) search for nonsystematized files on the official pages of the municipality; and (3) interviewing social actors about the importance of accessing data in understanding risks. The results showed that only $77 \%$ of the 84 items investigated had open files. All social actors claim to receive no incentives from the government to use open data, but consider making them available very important.

Keywords: risks and disasters, municipal management, Paraná

Editor responsável pela submissão: Julio Celso Borello Vargas.

Licenciado sob uma licença Creative Commons.

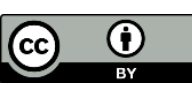

